\title{
Universiteit
}

Leiden

The Netherlands

\section{A quantitative evaluation of metallic conduction in conjugated polymers} Martens, H.C.F.; Brom, H.B.

\section{Citation}

Martens, H. C. F., \& Brom, H. B. (2004). A quantitative evaluation of metallic conduction in conjugated polymers. Physical Review B, 70(24), 241201. doi:10.1103/PhysRevB.70.241201

Version: $\quad$ Not Applicable (or Unknown)

License: $\quad$ Leiden University Non-exclusive license

Downloaded from: https://hdl.handle.net/1887/74792

Note: To cite this publication please use the final published version (if applicable). 


\title{
A quantitative evaluation of metallic conduction in conjugated polymers
}

\author{
H. C. F. Martens \\ Philips Research Laboratories, Prof. Holstlaan 4, 5656 AA Eindhoven, The Netherlands \\ H. B. Brom \\ Kamerlingh Onnes Laboratory, Leiden University, P.O. Box 9504, 2300 RA Leiden, The Netherlands
}

(Received 23 August 2004; published 2 December 2004)

\begin{abstract}
As the periodicity in crystalline materials creates the optimal condition for electronic delocalization, one might expect that in partially crystalline conjugated polymers delocalization is impeded by intergrain transport. However, for the best conducting polymers this presumption fails. Delocalization is obstructed by interchain rather than intergrain charge transfer and we propose a model of weakly coupled disordered chains to describe the physics near the metal-insulator transition. Our quantitative calculations match the outcome of recent broadband optical experiments and provide a consistent explanation of metallic conduction in polymers.
\end{abstract}

DOI: $10.1103 /$ PhysRevB.70.241201

PACS number(s): 72.15.Rn, 72.80.Le, 71.23.An, 71.30.+h

One of the hallmarks of metallic transport is the negative dielectric function $\varepsilon$ at low-frequency $\omega$, that reflects a time lag between induced current and applied field due to the inertia of delocalized charge carriers. The surprising observation of negative $\varepsilon$ in conjugated polymers by Kohlman and co-workers, ${ }^{1}$ which was recently verified by Romijn et al. and Lee and Heeger, ${ }^{2,3}$ spurred experimental and theoretical research into the nature of metallic conduction in these materials. As displayed in Fig. 1, the full spectral response is quite complicated including multiple zero crossings of $\varepsilon(\omega)$ and nonmonotonous behavior of the conductivity $\sigma(\omega)$. The low- $\omega$ dynamics are characterized by long scattering times $\tau \sim$ ps and low plasma frequencies $\omega_{\mathrm{p}} \sim \mathrm{meV}$. This differs three orders of magnitude from conventional metals where $\tau \sim$ fs and $\omega_{\mathrm{p}} \sim \mathrm{eV}$, while the carrier density $n$ is only one order of magnitude less. An empirical scaling relation between $\tau$ and $\omega_{\mathrm{p}}$ has been found (see Fig. 2), suggesting a common mechanism governs these parameters. ${ }^{4}$

The initial models for metallic polymers relied either on the Anderson theory of localization, ${ }^{5,6}$ or on a granular picture, ${ }^{1,5}$ but both predict $\varepsilon>0 .{ }^{6-9}$ Including percolation effects explains negative $\varepsilon,{ }^{1,9}$ but the calculated $\omega_{\mathrm{p}}$ lies two orders of magnitude above the experimental values. ${ }^{9}$ Prigodin and Epstein suggested that the metallic state is sustained by atypical resonant tunneling events and used the LandauerBüttiker transmission framework to explain small $\omega_{\mathrm{p}}$ and long $\tau .{ }^{10}$ However, the conditions for resonant tunneling are not always fulfilled, ${ }^{2}$ and the detailed physical mechanisms remain to be untangled.

Recently, Prigodin and co-workers successfully explained the $\omega$ dependence in the insulating phase using quasi-onedimensional (1D) variable-range hopping theory with interchain transfer as a rate-limiting step. ${ }^{11}$ By accounting for quasi-1D conduction, Kaiser et al. ${ }^{12}$ gave a description for metallic conductivity of granular polymers, which works for blends as well. ${ }^{13}$ Recent optical experiments by Lee and Heeger $^{3}$ and the present authors, ${ }^{4}$ could be explained in a 1D picture as well. Also Kohlman et al. discussed 1D effects. ${ }^{1}$ Here, we attempt to elucidate the nature of metallic conduction in polymers in more detail. We first argue why the me- tallic phase is dominated by the 1D structure. Then, as in Ref. 10, we use a Landauer-Büttiker approach to model the transport and show how interchain coupling drives the intrinsically insulating $1 \mathrm{D}$ phase to a metallic state at the crossover from 1D to three-dimensional (3D) coherent conduction. All transport parameters can be described in terms of disorder and coupling strength and the relevant length scales are $\zeta$ the 1D localization length, and $\lambda$ the length over which carriers experience sequential interchain events. For weak coupling $\zeta<\lambda$, transport keeps a 1D signature and effectively only a fraction of the carriers is involved in 3D transport. Our model reproduces the reported experimental results well and naturally ascribes the unusual low- $\omega$ dynamics to small interchain coupling.

In metallic conductors, delocalized states exist at the Fermi level. Delocalization is counteracted by disorder and the balance between metallic and insulating groundstates is critically dependent on the weakest charge transfer steps in the system. In the disordered polymers, chains are aligned within microcrystallites that are separated by amorphous regions, and delocalization may be impeded by either intergrain charge transfer or by interchain charge transfer. To identify the critical mechanism, we compare the energy scales.
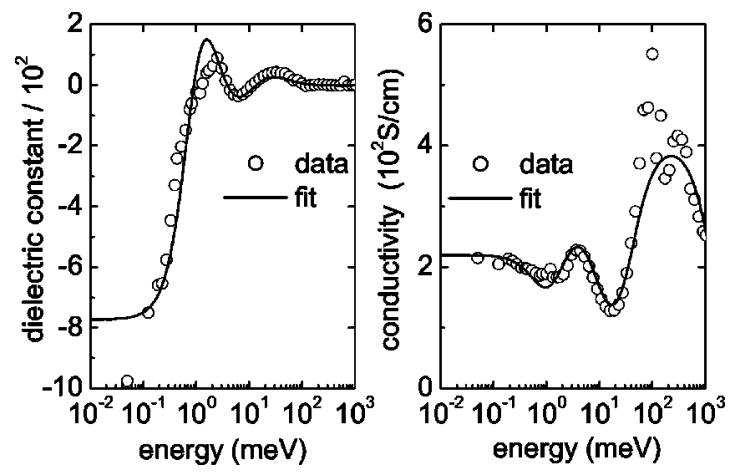

FIG. 1. Optical dielectric function and conductivity of metallic polypyrrole. Symbols: experimental results taken from Ref. 2. Drawn lines: theoretical prediction for weakly coupled disordered chains (see text). 
Intergrain charge transfer. For the best conducting polymers the crystalline fraction is $f_{c}=50 \%$ (polypyrrole, PPy, and polyaniline, PAni), even up to $f_{c}=90 \%$ for polyacetylene (PAc); the typical microcrystallite size $D_{c}$ amounts to $2 \mathrm{~nm}$ (PPy), $5 \mathrm{~nm}$ (PAni), or $10 \mathrm{~nm}$ (PAc). For isolated grains, ${ }^{10}$ geometrical considerations imply a width of amorphous regions $W_{a}=D_{c}\left(f_{c}^{-1 / 3}-1\right)$, giving $W_{a}=0.5,1.3$, and $0.4 \mathrm{~nm}$, respectively. Prigodin and Epstein estimate the localization length along the chain $\zeta=1.2 \mathrm{~nm} .{ }^{10} \mathrm{~A}$ different approach is based on the potential barrier height $B$ of the amorphous region, which from transport activation energy measurements we estimate $B=0.01-0.1 \mathrm{eV} .^{14} \mathrm{~A}$ wave function penetrating this barrier falls off exponentially with $\zeta=\left(2 m_{\mathrm{e}} B / \hbar^{2}\right)^{-1 / 2}=0.6-1.9 \mathrm{~nm}$. This corresponds to fully backscattered waves, expected for strong disorder, and agrees with the experimental value $\zeta=0.8 \mathrm{~nm}$ for insulating polythiophene. ${ }^{15}$ Another approach is based on a result by Thouless, $\zeta=a\left(E_{F} / B\right), 2,16$ which easily yields $10 \mathrm{~nm}$ using reasonable parameters $\left(E_{F}=0.5 \mathrm{eV}, B=0.1 \mathrm{eV}\right.$, and $a=4 \AA$ the microscopic length scale, e.g., a monomer). Taking a conservative estimate $\zeta=1.2 \mathrm{~nm}$, the intergrain transfer integral through amorphous regions along 1D chains, $I_{\|}=I_{0}$ $\times \exp \left(-2 W_{a} / \zeta\right)$, with $I_{0} \approx 2.5 \mathrm{eV}$ the intrachain $\pi$-electron transfer of the unperturbed chain, equals at least 1.1, 0.3, and $1.3 \mathrm{eV}$ for PPy, PAni, and PAc, respectively.

Interchain charge transfer. In conjugated polymers, the $\pi$-electron system is formed by hybridization of $2 p_{x}$ orbitals on adjacent carbon atoms that constitute the polymer backbone. Interchain charge transfer stems from the overlap of the $\pi$-electron clouds on neighboring chains. We estimated the interchain charge transfer $I_{\perp}$ for parallel chains of carbon $2 p_{x}$ orbitals using the Slater wave-function approximation. Our calculations suggest an optimum packing distance of $3 \AA$, where $I_{\perp} \approx 10 \mathrm{meV}$ is maximized. For larger separation, $I_{\perp}$ falls off exponentially with interchain localization length $\sim 1 \AA$, in agreement with experimental results. ${ }^{15}$ Extensive tight-binding calculations by Mizes and Conwell yielded $I_{\perp} \approx 30 \mathrm{meV}$ for PAc. ${ }^{17}$

Thus, for $f_{c} \approx 50 \%$ the energy scale for interchain charge transfer is at least an order of magnitude smaller than that for intergrain charge transfer. ${ }^{18}$ This is not surprising when it is realized that intergrain transport is essentially an intrachain process that proceeds along the covalently bound chains,

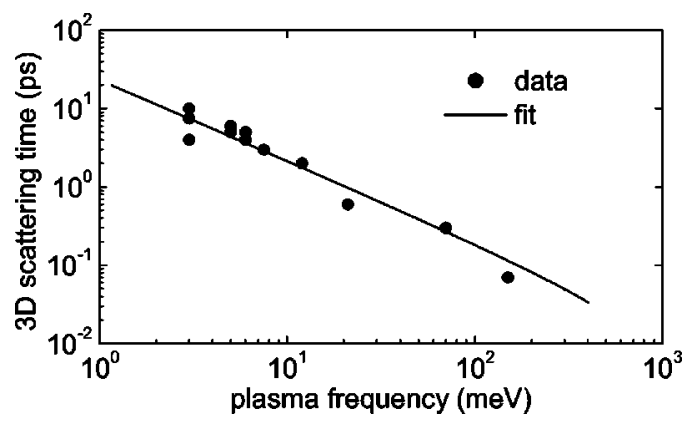

FIG. 2. Scaling relation between plasma frequency and 3D scattering time. Dots: experimental results taken from Ref. 4. Full line: calculation for disorder-driven metal-insulator transition (MIT) (see text). while adjacent chains are not chemically but Van der Waalsbonded and hence interchain electronic coupling should be much weaker. It means that (i) intrachain carrier delocalization extends over several grains and (ii) formation of truly delocalized states is governed by the small interchain charge transfer. Therefore, we propose a system of weakly coupled disordered chains to model conducting polymers. Our approach is as follows: we limit ourselves to an isolated disordered 1D chain where quantum mechanics leads to localized $1 \mathrm{D}$ wave functions, $\Psi_{1 \mathrm{D}}$. We argue that, staying within a $1 \mathrm{D}$ framework, interchain carrier exchange is equivalent to dephasing of the $\Psi_{1 \mathrm{D}}$ s and derive the condition for delocalization. Finally, we apply our model to describe the carrier dynamics of conducting polymers using known microscopic parameters and a tight-binding approximation for the interchain coupling.

First, we briefly review the case for isolated chains. Even for minimal disorder, the electronic eigenstates $\Psi_{1 \mathrm{D}}$ are localized due to repeated coherent backscattering. ${ }^{16}$ A convenient approach to solve the transport problem is the Landauer-Büttiker transmission framework. ${ }^{19}$ Assume a transmission coefficient $T \leqslant 1$ per unit length $a$. Then, over a distance $z>a$, the envelope of an unreflected wave falls off as $T^{z / a}$, implying an intrachain localization length $\zeta=a / \ln (1 / T)$. Disorder determines the microscopic transmission probabilities and hence sets $\zeta$. The mean level spacing of the localized states is $\Delta=1 /(g \zeta)$ with $g$ the density of states per unit length and unit energy. The dimensionless conductance $G=T /(1-T)$ and it follows that at short length scales $(z<\zeta) G$ is finite and given by the classical (Ohmic) result $G(z)=\zeta / z$, while for a long chain $G(z)=\exp (-z / \zeta)$ scaling is non-Ohmic and the conductivity, $G z$, becomes zero for $z \rightarrow \infty$. The conductivity due to resonant transitions between localized states is appreciable only for $\hbar \omega \sim \Delta$. For $\hbar \omega>\Delta$, this Lorentz oscillator response approaches the Drude conductivity with plasma frequency $\Omega_{\mathrm{p}} \sim \mathrm{eV}$ and intrachain scattering time $\tau_{0} \sim$ fs.

The above discussion is essentially based on a oneelectron Schrödinger equation assuming phase-coherent transport along the chain. Interchain transfer introduces a probability $\epsilon$ for interchain transitions and formally the isolated chain eigenfunctions are no longer appropriate. For small $\epsilon$, however, the isolated chain picture remains accurate for short time and length scales: the $\Psi_{1 \mathrm{D}}$ 's obtain a finite lifetime. In case of strong coupling such a picture is not realistic and the carrier transport problem should be considered for the fully coherent 3D system. In conjugated polymers, the interchain charge transfer is weak and a 1D transport model including the effect of coupling seems a good starting point. Particle conservation implies that interchain coupling occurs in the form of carrier exchange with a reservoir, where the carrier's initial state $\Psi_{1 \mathrm{D}, 1}$ differs from the final state $\Psi_{1 \mathrm{D}, 2}$, which thus introduces the finite lifetime of the 1D states: interchain transport leads to dephasing reflecting that the $\Psi_{1 \mathrm{D}}$ 's are not "true eigenstates" of a system of coupled chains. Usually, phase breaking is associated with inelastic events occurring at nonzero temperature. However, phase breaking is not necessarily dissipative when the initial and final states have the same energy. ${ }^{20}$ Allowing "elastic 
phase breaking" implies that the true eigenstates are extended over the entire system of coupled chains since then the energy level spacing $b^{2} /\left(g L^{3}\right)$ vanishes for $L \rightarrow \infty$ ( $b$ interchain distance), i.e., the system is gapless, or in other words, a metal. The condition for which this becomes possible determines the zero-temperature MIT and will be discussed further below.

Phase-breaking events are modeled in the LandauerBüttiker framework by incorporating current-conserving phase-randomizing scatterers into the chain. Each scatterer randomizes phase with amplitude $\sqrt{\epsilon}$, yielding probability $\epsilon$ for phase breaking. The total transmission probability is given by $T_{\mathrm{tot}}=T_{\mathrm{c}}+T_{\mathrm{i}},{ }^{19}$ with $T_{\mathrm{c}}$ the direct coherent intrachain transmission probability (no interchain events) and $T_{\mathrm{i}}$ transmission including at least one interchain transition. The conductance

$$
G=\frac{T_{\text {tot }}}{1-T_{\text {tot }}}=\frac{T_{c}}{1-T_{c}-T_{i}}+\frac{T_{i}}{1-T_{\mathrm{c}}-T_{i}}
$$

consists of two contributions: proportional to $T_{\mathrm{c}}$ and $T_{\mathrm{i}}$, respectively. However, the relations are not straightforward, as the denominators contain both $T_{\mathrm{c}}$ and $T_{\mathrm{i}}$.

The carrier fraction $\epsilon$ that suffered dephasing at $z=0$ will, on average, suffer a subsequent dephasing event at $z \sim \lambda=a / \epsilon$. We can discern two limits: $z<\lambda$ and $z>\lambda$. When $z<\lambda, T_{\mathrm{c}} \gg T_{\mathrm{i}} \sim \mathcal{O}(\epsilon)$, Eq. (1) simplifies to that of the isolated chain, $G=T_{\mathrm{c}} /\left(1-T_{\mathrm{c}}\right)$. When $z>\lambda, T_{\mathrm{c}}$ is exponentially small and $G=T_{\mathrm{i}} /\left(1-T_{\mathrm{i}}\right)$. Strong coupling, i.e., every scatterer dephases $(\epsilon=1), \lambda=\zeta$, corresponds to the classical (incoherent) case, $G=\lambda / z=\zeta / z$. For strong disorder and weak coupling, $\lambda>\zeta$, the transmission amplitude $T_{\mathrm{i}}$ becomes suppressed by localization effects. Then, $T_{\mathrm{i}}$ consists of the product of the dephasing probability $\epsilon$ and a factor $\zeta / a$ that counts the number of channels available for the interchain transport on the scale $\zeta$, where the wave functions have appreciable amplitude, multiplied by $\exp (-\lambda / \zeta)$, the $1 \mathrm{D}$ coherent transmission amplitude between subsequent dephasing events, yielding $G=\epsilon(\zeta / a)(\lambda / z) \exp (-\lambda / \zeta)=(\zeta / z) \exp (-\lambda / \zeta)$. Thus, for weak coupling, the classical expectation value for the conductance is suppressed by 1D localization effects. Delocalized carrier transport remains piecewise 1D and the length scale of interest is $\lambda$ with a 1D transport time $\tau_{\lambda}=\left(\zeta / v_{\mathrm{F}}\right) \exp (\lambda / \zeta)$. At $\tau_{\lambda}$ scale, only fraction $\epsilon$ of the carriers is involved in interchain transport and thus crossover to full 3D conduction occurs at $\tau_{3 \mathrm{D}}=\tau_{\lambda} / \epsilon$.

The above results demonstrate that in the presence of dephasing, the dc conductivity remains finite for $z \rightarrow \infty$. This holds for any dephasing mechanism, in particular, inelastic events due to finite temperature. ${ }^{21}$ As argued above, "elastic dephasing" is a necessary condition for the formation of a truly metallic state. In our 1D model, the interchain transfer couples a $\Psi_{1 \mathrm{D}, 1}$ to a different $\Psi_{1 \mathrm{D}, 2}$ on length scale $\lambda$, and this gives a typical level splitting $\epsilon \Delta$ of these states. When the finite width $h / \tau_{\lambda}$ of the 1D energy levels on length scale $\lambda$ (Thouless energy) exceeds the level splitting $\epsilon \Delta$, energy matched (elastic) interchain transitions are possible and hence a metallic phase can be formed. The inset of Fig. 3 schematically indicates this mechanism. Applying the rela-

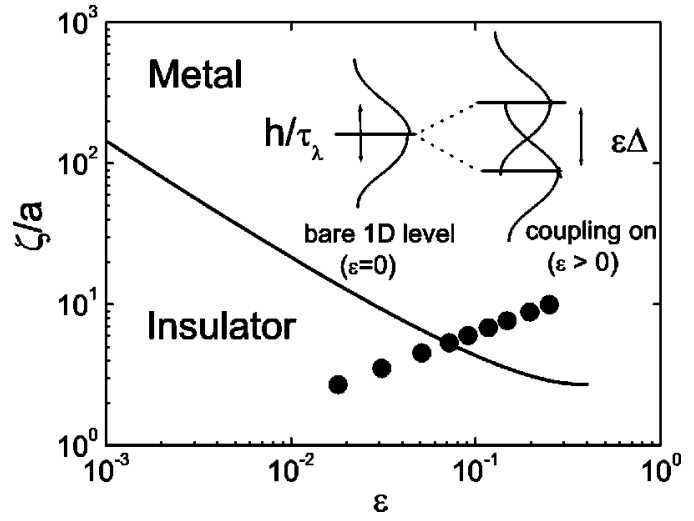

FIG. 3. Metal-insulator phase diagram of coupled 1D chains as a function of interchain coupling and intrachain localization length. Below the drawn line the system is insulating, above it is metallic. Dots: disorder-driven MIT in conducting polymers. The inset gives a graphical representation of the leitmotif behind the MIT criterion discussed in the text.

tion $h v_{\mathrm{F}}=g^{-1}$ valid for 1D conductors, the criterion for delocalization translates into $a / \zeta=\left(B / E_{\mathrm{F}}\right)^{2}<\epsilon \ln (1 / \epsilon)$. Alternatively, one can use the condition $G>1$ for a metal on a characteristic length scale. When applied to the carrier fraction that is effectively involved in the interchain transport on length scale $\lambda$, this gives $G_{\epsilon}(\lambda)=G(\lambda) / \epsilon=\exp (-\lambda / \zeta) / \epsilon>1$, leading to the same result. The phase diagram for coupled disordered chains as function of disorder $(\zeta / a)$ and interchain coupling $(\epsilon)$ is shown in Fig. 3.

Let us now apply our model to the metallic polymers. First we derive $\epsilon$ using a tight-binding approximation. Interchain charge transfer would, in the absence of disorder, lead to an interchain bandwidth $\propto I_{\perp}$. However, as a result of disorder, electronic states $\Psi_{1 \mathrm{D}}$ on adjacent chains typically have an energy mismatch $\approx \Delta / 2$. Provided that $I_{\perp}<\Delta / 2$, the bandwidth available for interchain transport reduces to $4 I_{\perp}^{2} / \Delta$, which corresponds to an interchain coupling $\epsilon=4\left(I_{\perp} / \Delta\right)^{2}$ governed by both interchain transfer and disorder. Next, we derive the relevant transport time (energy) scales. Crossover to full 3D conduction occurs on a time scale $\tau_{3 \mathrm{D}}=\tau_{\lambda} / \epsilon$. Following the reasoning for the MIT criterion, the bandwidth for interchain transport is $\epsilon \Delta \exp (-\lambda / \zeta)$ and, using tight binding, the effective mass for 3D transport becomes $m^{*}=8 \hbar^{2} \exp (\lambda / \zeta) /\left(\epsilon \Delta b^{2}\right)$. 1D localization suppresses the transmission probability on scale $\lambda$ and the effective carrier density involved in 3D transport reduces to $n_{\text {eff }}=n \exp (-\lambda / \zeta)$. This gives

$$
\begin{gathered}
\tau_{3 \mathrm{D}}=\left(\pi \hbar / 2 I_{\perp}^{2} g \zeta\right) \exp \left(a / 4 I_{\perp}^{2} g^{2} \zeta^{3}\right), \\
\omega_{\mathrm{P}}=\sqrt{n e^{2} b^{2} I_{\perp}^{2} g \zeta / 2 \hbar^{2} \varepsilon_{0}} \exp \left(-a / 4 I_{\perp}^{2} g^{2} \zeta^{3}\right) .
\end{gathered}
$$

For $\omega \tau_{3 \mathrm{D}}<1$, the $3 \mathrm{D}$ transport channel is open and the conductivity is $\sigma(\omega)=\sigma_{3 \mathrm{D}} /\left(1+i \omega \tau_{3 \mathrm{D}}\right)$. At intermediate energy, $\hbar / \tau_{3 \mathrm{D}}<\hbar \omega<\Delta$, the $1 \mathrm{D}$ signature of delocalized carrier transport becomes visible. Screening in 1D conductors is poor and the dynamic response is described as $\sigma(\omega)=\sigma_{\mathrm{ni}}(\omega) /\left[1+\sigma_{\mathrm{ni}}(\omega) /(i \omega C)\right], \quad$ where $\quad \sigma_{\mathrm{ni}}(\omega)=\sigma_{\lambda} /(1$ 
$\left.+i \omega \tau_{\lambda}\right)$, the noninteracting case, and capacitance $C \approx e^{2} g$ quantifies the $1 \mathrm{D}$ screening efficiency. ${ }^{22}$ For $\hbar \omega>\Delta$ the isolated chain response (Lorentz oscillator) is obtained: $\sigma(\omega)=\sigma_{0} /\left\{1+i\left[\left(\omega^{2}-\omega_{0}^{2}\right) / \omega\right] \tau_{0}\right\}$, with $\omega_{0}$ a measure for level separation. In case the time scales are sufficiently separated, the total optical response is

$$
\sigma(\omega) \approx \frac{\sigma_{3 \mathrm{D}}}{1+i \omega \tau_{3 \mathrm{D}}}+\frac{\sigma_{\lambda}}{1+i \omega \tau_{\lambda}+\frac{\sigma_{\lambda}}{i \omega C}}+\frac{\sigma_{0}}{1+i \frac{\omega^{2}-\omega_{0}^{2}}{\omega} \tau_{0}}
$$

Here $\sigma_{3 \mathrm{D}}=\varepsilon_{0} \omega_{\mathrm{p}}^{2} \tau_{3 \mathrm{D}}, \quad \sigma_{\lambda}=\sigma_{3 \mathrm{D}}$ because the factors $\epsilon$ in $\tau_{\lambda}=\epsilon \tau_{3 \mathrm{D}}$ and $\omega_{\mathrm{p}, \lambda}^{2}=\omega_{\mathrm{p}}^{2} / \epsilon$ [proportional to bandwidth $\Delta \exp (-\lambda / \zeta)]$ cancel, and $\sigma_{0}=\varepsilon_{0} \Omega_{\mathrm{p}}^{2} \tau_{0}=n e^{2} \tau_{0} / m$ is the intrinsic intrachain conductivity. Indeed, Fig. 1 demonstrates our theory and gives an excellent description of the experimental results, using $\tau_{0}=3 \mathrm{fs}, \tau_{\lambda}=0.4 \mathrm{ps}, \tau_{3 \mathrm{D}}=5 \mathrm{ps}$, $\sigma_{3 \mathrm{D}}=\sigma_{\lambda}=220 \mathrm{~S} / \mathrm{cm}, \quad \sigma_{0}=380 \mathrm{~S} \mathrm{~cm}, \quad \omega_{0}=0.23 \mathrm{eV}, \quad$ and $C=10^{-10} \mathrm{~F} / \mathrm{m}$. Thus, $\lambda / \zeta=3.5$ and $\epsilon=0.08$, which, assuming $a=4 \AA$, leads to $\zeta=1.4 \mathrm{~nm}$ and $\lambda=5.0 \mathrm{~nm}$.

We conclude by addressing the scaling relation between $\omega_{\mathrm{p}}$ and $\tau_{3 \mathrm{D}}$. Equations (2) and (3) express the extreme sensitivity of the 3D carrier dynamics for disorder and interchain coupling. For increasing disorder (lower $\zeta$ ) and de- creasing coupling (lower $I_{\perp}$ ) the transition time to $3 \mathrm{D}$ conduction increases exponentially, while simultaneously the plasma frequency decreases at the same rate. Let us consider the case of a disorder-driven MIT. We chose $\tau_{0}=1.2-4.0 \mathrm{fs}$ as a typical range and $\zeta=4 v_{\mathrm{F}} \tau_{0}$ (Ref. 10) to reproduce the experimental results in Fig. 2 using known microscopic parameters for conducting polymers: ${ }^{5} I_{\perp}=50 \mathrm{meV}, a=4 \AA$, $b=3 \AA, g=0.5 \mathrm{eV}^{-1} a^{-1}$, and $n=7 \times 10^{27} \mathrm{~m}^{-3}$, which corresponds to optimum doping of 0.25 carrier per monomer. A similar calculation can be performed for a MIT purely driven by interchain transfer, but agreement with experiment can be achieved only for an unrealistically large change in $I_{\perp}$ of 20-90 meV. This agrees with the general consensus that the MIT in polymers is disorder driven. Using the above parameters, we have calculated the phase diagram for the disorderdriven MIT (see Fig. 3), illustrating the transition to the metallic state. Indeed it shows the conducting polymers are on the edge of being metallic or insulating.

In summary, we have discussed a transport model for weakly coupled disordered chains. Under appropriate conditions, $\left(B / E_{\mathrm{F}}\right)^{2}<\epsilon \ln (1 / \epsilon)$, a crossover from 1D to 3D transport occurs, and a metallic phase is formed. Expressions for the optical conductivity, 3D scattering time and plasma frequency have been derived. Based on established microscopic parameters, the model quantitatively explains broadband data on metallic PPy.
${ }^{1}$ R. S. Kohlman, A. Zibold, D. B. Tanner, G. G. Ihas, T. Ishiguro, Y. G. Min, A. G. MacDiarmid, and A. J. Epstein, Phys. Rev. Lett. 78, 3915 (1997).

${ }^{2}$ I. G. Romijn, H. J. Hupkes, H. C. F. Martens, H. B. Brom, A. K. Mukherjee, and R. Menon, Phys. Rev. Lett. 90, 176602 (2003).

${ }^{3}$ K. Lee and A. J. Heeger, Phys. Rev. B 68, 035201 (2003).

${ }^{4}$ H. C. F. Martens, J. A. Reedijk, H. B. Brom, D. M. de Leeuw, and R. Menon, Phys. Rev. B 63, 073203 (2001).

${ }^{5}$ R. Menon, C. O. Yoon, D. Moses, and A. J. Heeger, Handbook of Conducting Polymers, 2nd ed. (Marcel Dekker, New York, 1998).

${ }^{6}$ K. Lee, R. Menon, C. O. Yoon, and A. J. Heeger, Phys. Rev. B 52, 4779 (1995).

${ }^{7}$ B. Chapman, R. G. Buckley, N. T. Kemp, A. B. Kaiser, D. Beaglehole, and H. J. Trodahl, Phys. Rev. B 60, 13479 (1999).

${ }^{8}$ G. Tzamalis, N. A. Zaidi, C. C. Homes, and A. P. Monkman, Phys. Rev. B 66, 085202 (2002).

${ }^{9}$ O. Levy and D. Stroud, Phys. Rev. B 56, 8035 (1997).
${ }^{10}$ V. N. Prigodin and A. J. Epstein, Synth. Met. 125, 43 (2002).

${ }^{11}$ V. N. Prigodin, A. N. Samukhin, and A. J. Epstein, Synth. Met. 141, 155 (2004).

${ }^{12}$ A. B. Kaiser, Adv. Mater. (Weinheim, Ger.) 13, 927 (2001).

${ }^{13}$ Y. Long, Z. Chen, N. Wang, J. Li, and M. Wan, Physica B 334, 82 (2004).

${ }^{14}$ H. C. F. Martens, H. B. Brom, and R. Menon, Phys. Rev. B 64, 201102(R) (2001); 65, 079901 (2002).

${ }^{15}$ J. A. Reedijk, H. C. F. Martens, H. B. Brom, and M. A. J. Michels, Phys. Rev. Lett. 83, 3904 (1999).

${ }^{16}$ D. J. Thouless, J. Phys. C 6, L49 (1973).

${ }^{17}$ H. A. Mizes and E. M. Conwell, Phys. Rev. B 43, 9053 (1991).

${ }^{18}$ For $f_{c} \leqslant 25 \%$ intergrain transport limits delocalization.

${ }^{19}$ M. Büttiker, IBM J. Res. Dev. 32, 63 (1988).

${ }^{20}$ S. Datta, J. Phys.: Condens. Matter 2, 8023 (1990).

${ }^{21}$ K. Maschke and M. Schreiber, Phys. Rev. B 49, 2295 (1994).

${ }^{22}$ M. Büttiker, A. Prêtre, and H. Thomas, Phys. Rev. Lett. 70, 4114 (1993). 